finger nails, on skin, clothes, as well as fecal-oral spread. In patients without known contacts, restaurant food handlers from endemic areas are suspected as a source of infection. Neurocysticercosis and serological testing must be added to the differential diagnosis of a seizure disorder in children.

\title{
MRI IN SUBACUTE SCLEROSING PANENCEPHALITIS
}

MRI studies in 26 patients, ages 3 to 25 years, with subacute sclerosing panencephalitis (SSPE) are reported from the Departments of Pediatric Neurology and Radiology, Hacettepe University, and SSK Ankara Hospital, Ankara, Turkey. Lesions of high signal intensity on T2-weighted images were present in the cerebral cortex and subcortical and periventricular white matter. A local mass effect occurred in 5 patients. White matter involvement extended to the splenium of the corpus callosum, whereas basal ganglia, thalamic nuclei, and brainstem were spared. Parenchymal lesions had a weak correlation with clinical symptoms and signs, especially motor and visual symptoms. Periventricular lesions and cerebral atrophy showed no clinical correlation. (Anlar B, Saatci I, Kose G, Yalaz K. MRI findings in subacute sclerosing panencephalitis. Neurology Nov 1996;47:1278-1283). (Reprints: Dr Banu Anlar, Hacettepe University Faculty of Medicine, Department of Pediatric Neurology, Ankara 06100, Turkey).

COMMENT. The MRI may be helpful in the diagnosis of SSPE. The presenting symptoms are usually myoclonic jerks and mental deterioration. A positive titer of measles antibodies in the CSF and a characteristic EEG are additional manifestations.

\section{TRAUMATIC DISORDERS}

\section{FETAL CNS DAMAGE WITH MATERNAL PRENATAL TRAUMA}

The neurologic findings in nine infants who were exposed to maternal trauma during pregnancy are reported from the University Hospital Essen, Hufelandstr, Germany. Motor-vehicle accidents occurred in 7 and blunt abdominal trauma in 2 . One infant required resuscitation and one premature needed assisted ventilation. Movement disorders occurred in 3, hemiparesis in 2 , convulsions (1), and 3 were normal. Neuroimaging showed periventricular leukomalacia in 2, localized vascular infarction in 2, hemorrhage (1), hydrocephalus (2), and diffuse brain damage (1). The relation of the cerebral damage to the maternal trauma was certain in one child and probable in the remainder. (Baethmann M, Kahn T, Lenard H-G, Voit T. Fetal CNS damage after exposure to maternal trauma during pregnancy. Acta Paediatr Nov 1996;85:1331-1338). (Respond: Dr Martina Baethmann, Department of Pediatrics, University Hospital Essen, Hufelandstr, 55, 45122, Essen, Germany).

COMMENT. Maternal trauma during pregnancy may result in fetal cerebral damage that manifests with neurologic symptoms in surviving infants. Mothers should be examined carefully for signs of premature labor after trauma, and the newborn infant monitored for neurologic damage. Maternal acidosis is found to correlate with severity of fetal damage and mortality. 